\title{
Action of bleomycin is affected by bleomycin hydrolase but not by caveolin-1
}

\author{
JIANGUO CHEN, YANG CHEN and QIYANG HE \\ Institute of Medicinal Biotechnology, Peking Union Medical College and \\ Chinese Academy of Medical Sciences, Beijing 100050, P.R. China \\ Received July 25, 2012; Accepted October 1, 2012
}

DOI: $10.3892 /$ ijo.2012.1668

\begin{abstract}
Bleomycin is a first-line clinically used antitumor antibiotic for effective treatment of certain types of cancer in combination with other antitumor agents. Its action is affected by bleomycin hydrolase (BLH), DNA repair enzymes, membrane transport proteins and other cellular factors. To clarify whether BLH confers the resistance to bleomycin in tumor cells, it is necessary to further investigate the roles of BLH and its combination with other factors such as caveolin-1 in the action of bleomycin. In this study fourteen human cell lines were used for determination of bleomycin action and roles of BLH and caveolin-1. The relationship between action of bleomycin and cellular amount of BLH was detected by the MTT method and western blotting in the human leukemia cell line HL-60, HeLa cervical cancer cells and $\mathrm{HaCaT}$ immortalized keratinocyte cells. The sensitivity to bleomycin was increased in HeLa cells after knockdown of BLH mRNA by RNA interference. There is no relationship between caveolin-1 levels and action of bleomycin, although the distribution of the cell cycle was altered in the caveolin-1-knockdown HeLa cells after treatment with bleomycin. In addition, regulation of BLH and caveolin-1 expression in HeLa and HaCaT cells was observed in a concentration-dependent manner after exposure to bleomycin. In conclusion, bleomycin hydrolase is one of the biomarkers for determination of bleomycin action. Although caveolin-1 can respond to bleomycin treatment, it is unrelated to bleomycin sensitivity.
\end{abstract}

\section{Introduction}

Bleomycin is first line clinically used antitumor antibiotic, especially in combination with other antitumor agents for effective treatment of several malignant tumors including squamous cell carcinoma, malignant lymphoma and testicular cancer. It exerts its antitumor effects through a sequence-selective, iron-

Correspondence to: Dr Qiyang He, Institute of Medicinal Biotechnology, Peking Union Medical College and Chinese Academy of Medical Sciences, No. 1 Tiantan Xili, Beijing 100050, P.R. China E-mail: qyh2000bj@yahoo.com.cn

Key words: bleomycin hydrolase, bleomycin, caveolin-1, biomarker dependent oxidative cleavage of DNA and RNA in the presence of oxygen and finally initiation of the cell death program, such as apoptosis and cellular senescence (1). However, its side actions such as inducing pulmonary fibrosis and flagellate hyperpigmentation retard further application in clinic $(2,3)$. Attempts have been made to develop new superior bleomycin antibiotics to improve antitumor activity and overcome the drawbacks of severe pulmonary toxicity. For instance, the new antibiotic boningmycin, a member of bleomycin family, was purified by our institute from the fermentation broth of Streptomyces verticillus var. pingyangensis $n . s p$ in China. It exhibits antibacterial activities and stronger antitumor activities with lower pulmonary toxicity $(4,5)$.

For the translational development of new bleomycin antibiotic, it is necessary to investigate the biomarkers for prediction of bleomycin sensitivity in tumor cells. Action of bleomycin is affected by bleomycin hydrolase (BLH), DNA repair enzymes, membrane transport proteins, antioxidant enzymes and other cellular factors. Previous studies have confirmed that BLH can hydrolyze the bleomycin to generate the inactive deamino form (6). The mutant mice lacking BLH gene were very sensitive to bleomycin (7). Expression of the yeast BLH gene in the mouse NIH3T3 cells resulted in nearly 5-fold increased resistance to bleomycin (8). Therefore, lower expression of BLH in skin and lung is thought to effectively treat head-neck carcinoma and lead to severe pulmonary toxicity $(1,2,9)$. However, whether BLH can confer the resistance to bleomycin remains controversial. BLH expression level was not well correlated with the resistance to bleomycin in the human bleomycin-resistant cell lines (10). The resistance to bleomycin in yeast was not conferred by overexpression of yeast BLH (11).

Bleomycin action is tissue specific. In clinic chemotherapy for testicular germ cancer, the regimen consisting of bleomycin, cisplatin and etoposide plus surgery techniques has markedly inproved the survuval rates from $5 \%$ in 1970 s to $80 \%$ in 2000 (12). It may be mainly contributed by bleomycin as a recent study showed that carnitine transporter hCT2 encoded by the SLC22A16 gene efficiently transferred bleomycin-A5 into the NT2/D1 human testicular cancer cells, leading to extreme sensitivity to bleomycin-A5 (13).

Besides the above mentioned molecules, the action of bleomycin may be affected by other cellular factors such as caveolin-1 (Cav-1). Knockdown of Cav-1 prevented bleomycininduced growth arrest at the $\mathrm{G}_{2} / \mathrm{M}$ phase (14). Bleomycin 
treatment of A549 cells resulted in an increased interaction between Cav-1 and MGr1-Ag and a slight enrichment of MGrl-Ag in lipid rafts (15). Overexpression of Cav-l suppressed bleomycin-induced pulmonary fibrosis in the mice (16). These results indicate that Cav-1 may contribute to the resistance to bleomycin.

In order to explore the biomarkers for determination of bleomycin action, we further evaluated the roles of BLH and cav-1 to bleomycin in the different cell lines. The results showed that BLH conferred the natural resistance to bleomycin in some type of cell lines.

\section{Materials and methods}

Drugs and chemicals. Bleomycin, dimethyl sulfoxide, 3-(4,5-dimethyl-2-thiazoyl)-2,5-diphenyl-2H-tetrazolium bromide (MTT) and propidium iodide were purchased from Sigma-Aldrich Chemical Inc. (St. Louis, MO, USA). DMEM, RPMI-1640, Lipofectamine ${ }^{\mathrm{TM}} 2000$ and Stealth RNAi ${ }^{\mathrm{TM}}$ siRNA (scrambled siRNA) were obtained from Invitrogen (Carlsbad, CA, USA).

Cell lines and culture. Human hepatoma HepG2 cells, human non-small lung cancer A549 cells, human promyelocytic leukemia HL-60 cells and human cervical cancer HeLa cells were purchased from the American Type Culture Collection (Rockville, MD, USA). Human immortalized keratinocyte $\mathrm{HaCaT}$ cells were purchased from Yonsei University (Korea). The normal human hepatocyte L02 cells was purchased from the Institute of Cell Biology (Shanghai, China). The normal human embryo liver HEL cells and human embryo lung fibroblast HPF cells were purchased from Institute of Basic Medical Sciences, Chinese Academy of Medical Sciences (Beijing, China). The cell lines were cultured in RPMI-1640 or DMEM medium supplemented with $10 \%$ fetal bovine serum, $100 \mathrm{U} /$ $\mathrm{ml}$ penicillin and $100 \mu \mathrm{g} / \mathrm{ml}$ streptomycin. All cultures were maintained in an incubator at $37^{\circ} \mathrm{C}$ with $5 \% \mathrm{CO}_{2}$ in a humidified atmosphere.

Cytotoxicity assays. Cell viability was assessed using the MTT assay. The cells were seeded into a 96-well plate at a cell density of 3000 per well for $24 \mathrm{~h}$, followed by drug treatment for $72 \mathrm{~h}$. Next, $5 \mu 1$ of $5 \mathrm{mg} / \mathrm{ml}$ MTT was added to the medium and incubated for $2 \mathrm{~h}$ at $37^{\circ} \mathrm{C}$. After removing the culture medium, $150 \mu \mathrm{l}$ of dimethyl sulfoxide was added. The plates were read using an enzyme-linked immunosorbent assay plate reader at $570 \mathrm{~nm}$. The viability of untreated cells was set as $100 \%$ and the viability in other groups was calculated by comparing the optical density reading with the control. $\mathrm{IC}_{50}$ value was calculated from the nonlinear regression analysis.

Cell cycle analysis by flow cytometry. The cells were trypsinized and washed once with PBS, and then fixed with cold 70\% ethanol overnight. The fixed cells were washed twice with PBS and incubated with $100 \mu \mathrm{g} / \mathrm{ml}$ of ribonuclease A at $37^{\circ} \mathrm{C}$ for $30 \mathrm{~min}$ and then stained in PBS containing $50 \mu \mathrm{g} / \mathrm{ml}$ propidium iodide for $1 \mathrm{~h}$. The fluorescence intensity was detected using BD FACSCalibur cytometer (BD Biosciences, CA, USA) and the cell cycle distribution was assayed using the ModFit LT software (BD Biosciences).
Reverse transcriptase-PCR (RT-PCR). The RNA (500 ng) was reverse-transcribed with the Moloney murine leukemia virus reverse transcriptase (Promega Corp., Madison, WI, USA) and oligo(dT) 16 primer (Promega) in $20 \mu \mathrm{l}$ of reaction mixture. The resulting cDNA (1-2 $\mu \mathrm{l})$ was amplified by PCR for BLH and glyceraldehyde-3-phosphate dehydrogenase. cDNA was amplified in 28-32 cycles, consisting of denaturing for $30 \mathrm{sec}$ at $94^{\circ} \mathrm{C}$, annealing for $45 \mathrm{sec}$ at $50^{\circ} \mathrm{C}$, and primer extension for $30 \mathrm{sec}$ at $72^{\circ} \mathrm{C}$. BLH forward primer: ACCAGCCCATTGACT TCC and reverse primer: TGTCCACCACCACTTCGT, glyceraldehyde-3-phosphate dehydrogenase forward primer: CGG AGTCAACGGATTTGGTCGTAT and reverse primer: AGCC TTCTCCATGGTGGTGAAGAC were used for RT-PCR.

Western blot analysis. Cells were lysed with the lysis buffer containing $50 \mathrm{mmol} / \mathrm{l}$ Tris- $\mathrm{HCl}(\mathrm{pH} 7.5), 250 \mathrm{mmol} / \mathrm{l} \mathrm{NaCl}$, $5 \mathrm{mmol} / \mathrm{l}$ EDTA, $50 \mathrm{mmol} / \mathrm{l} \mathrm{NaF}, 1 \mathrm{mmol} / \mathrm{l} \mathrm{DTT}, 1 \%$ Triton $\mathrm{X}-100,1 \mathrm{mmol} / \mathrm{l}$ sodium orthovanadate, and protease inhibitors. After centrifugation, the supernatant fraction was removed and protein concentrations were determined using the Bio-Rad protein assay. Proteins were resolved by SDS-PAGE and transferred onto a polyvinylidene fluoride membrane. After blocking with 5\% non-fat milk in the blocking buffer (PBS containing $0.1 \%$ Tween-20, $\mathrm{pH} 7.5$ ), the membrane was incubated with the desired primary antibody for $2 \mathrm{~h}$ at room temperature and then incubated with an appropriate peroxidase-conjugated secondary antibody. The immunoreactive bands were visualized using the ECL Plus Western Blotting Detection System (Piscataway, NJ, USA). The level of $\beta$-actin was used as a loading control. The antibody against Cav-1 were from Cell Signaling Technology, Inc. (Danvers, MA, USA). The antibodies against actin and BLH were from Santa Cruz Biotechnology, Inc. (Santa Cruz, CA, USA).

RNA interference. Cells were transfected with Lipofectamine 2000 (Invitrogen) according to the manufacturer's instructions using the double-stranded small interfering RNA (siRNA) oligonucleotides (100 pmol siRNA for each gene). Cell treatments were started $24 \mathrm{~h}$ after plating. BLH siRNA-3: 5'-UACC CAAACAGAUGCACACCACUCG-3'. Cav-1 siRNA-2: 5'-UU UCCCAACAGCUUCAAAGAGUGGG-3'.

Statistical analysis. Data were expressed as means with standard deviations. One-way analysis of variance (ANOVA) was used to analyze the variance for the means of multiple groups. Statistical analysis was performed using SPSS 12.0, and significance was accepted at $\mathrm{P}<0.01$.

\section{Results}

Different sensitivities to bleomycin in the cells with high levels of $B L H$ or $C a v-1$. To explore the relationship between BLH and Cav-1 with bleomycin action, we examined the expression levels of BLH and Cav-1 in fourteen different human cell lines and three distinct cell lines HL-60, HaCaT and HeLa were selected for the further experiment. Western blot analysis confirmed that BLH was indeed strongly expressed in HL-60 cells, whereas its expression was undetectable in $\mathrm{HaCaT}$ cells (Fig. 1A and B). By contrast, high Cav-1 amount was detected in $\mathrm{HaCaT}$ cells (Fig. $1 \mathrm{~A}$ and $\mathrm{C}$ ). 


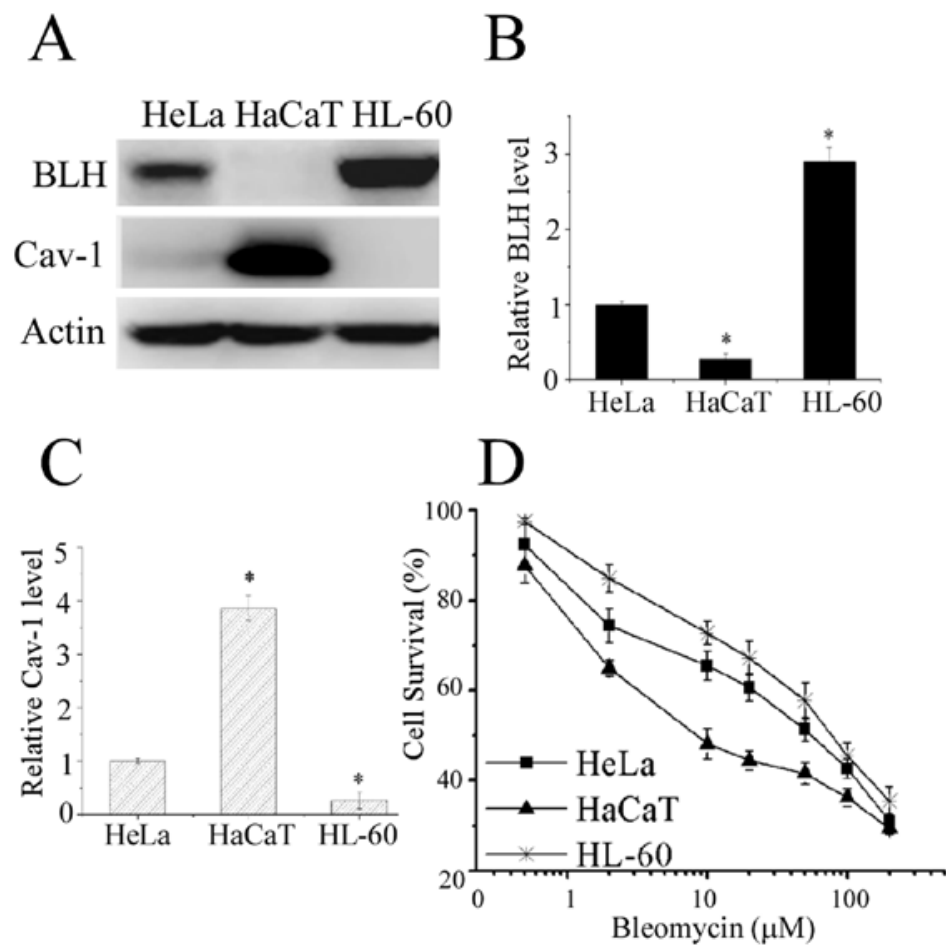

Figure 1. Sensitivity to bleomycin with cellular BLH or Cav-1. (A) The expression levels of BLH and Cav-1 in the HeLa, HaCaT and HL-60 cells were detected by western blotting. The levels of BLH (B) and Cav-1 (C) were quantified. (D) The cell survival curves of HeLa, HaCaT and HL-60 cells after exposure to bleomycin for $2 \mathrm{~h}$, washing out of the drug and then incubation for $72 \mathrm{~h}$. The cell viabilities were determined by MTT assay. Results are expressed as the mean \pm SD from three separate experiments. ${ }^{*} \mathrm{P}<0.01$ vs the respective control.
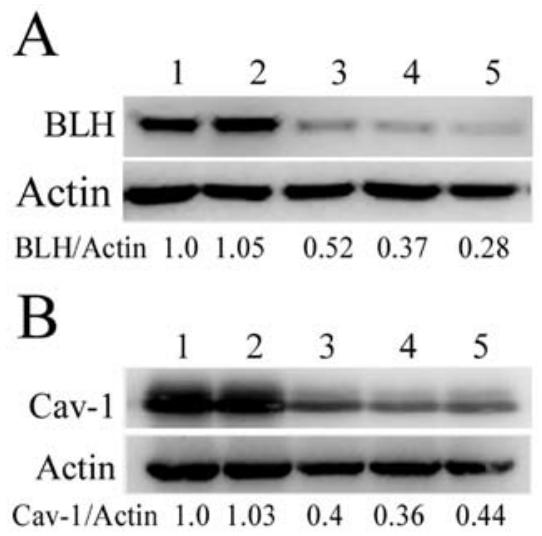

Figure 2. Knockdown of BLH and Cav-1 expression by siRNA. (A) BLH and (B) Cav-1, the lanes are control (1), scrambled control siRNA (2), siRNA-1 (3), siRNA-2 (4) and siRNA-3 (5). One representative result from three independent experiments is shown.

The sensitivity to bleomycin in these cells were determined by MTT assay and plotted on the survival curves (Fig. 1D). The $\mathrm{IC}_{50}$ values for HaCaT, HeLa and HL-60 cells were 13.1, 48.2 and $65.8 \mu \mathrm{M}$, respectively. HaCaT cells devoid of detectable BLH expression was the most sensitive to bleomycin, suggesting a liner relationship with BLH amount. To further confirm the correlation between BLH or Cav-1 expression and sensitivity to bleomycin, we assessed it by knockdown of BLH or Cav-1 with RNA interference in the cells.

Knockdown of BLH and Cav-1 expression by RNA interference. We designed three different BLH or Cav-1siRNAs targeted to
BLH and Cav-1 mRNA, respectively. The levels of BLH and Cav-1 were analyzed by western blotting after HeLa and $\mathrm{HaCaT}$ cells were transfected with siRNAs for $72 \mathrm{~h}$. They were obviously reduced, whereas transfection with scrambled siRNA had no effect (Fig. 2). The BLH siRNA-3 and Cav-1 siRNA-2 with most efficient inhibition were used for the following experiments.

Reduction of bleomycin action by knockdown of BLH. To further evaluate whether BLH was involved in modulating bleomycin action, we assessed the sensitivity of HeLa cells to bleomycin following knockdown of BLH with siRNA specific to BLH. The inhibition of BLH mRNA and BLH protein expression reached 73 and $80 \%$, respectively (Fig. 3A). The effect of BLH knockdown with respect to bleomycin action was performed by MTT assay. As illustrated in Fig. 3B, the $\mathrm{IC}_{50}$ values of control, scrambled siRNA and BLH siRNA group were 3.63, 3.34 and $0.82 \mu \mathrm{M}$, respectively, after exposure to bleomycin for $72 \mathrm{~h}$. The sensitivity to bleomycin increased 3.4-fold after knockdown of $\mathrm{BLH}$, confirming involvement of BLH in modulating bleomycin action.

Knockdown of Cav-1 was unrelated with sensitivity to bleomycin. To determine whether Cav-1 conferred resistance to bleomycin, Cav-1 siRNA was used for knockdown of Cav-1. Effective reductions of Cav-1 level were detected by western blot analyses (Fig. 4A and C). Illustrated in Fig. 4B and D, knockdown of Cav-1 did not alter the inhibitory actions of bleomycin towards HeLa and $\mathrm{HaCaT}$ cells, indicating that Cav-1 is not involved in the sensitivity to bleomycin. 

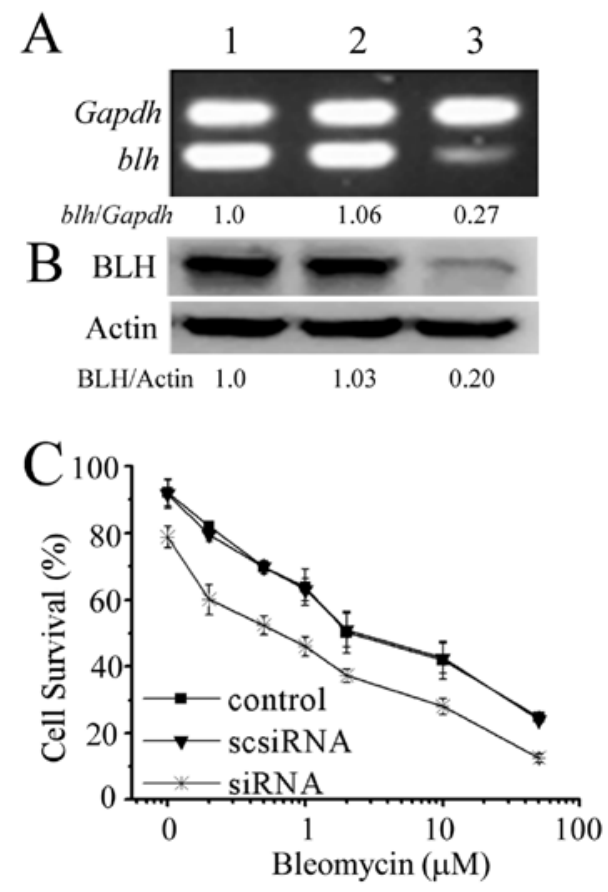

Figure 3. Reduction of bleomycin action by knockdown of BLH. The levels of BLH mRNA (A) and BLH protein (B) were reduced after transfection of HeLa cells with siRNAs. Lane 1, control; lane 2, scrambled control siRNA; lane 3, BLH siRNA. (C) The cell viabilities were determined by MTT assay after knockdown of BLH and then treatment with bleomycin for $72 \mathrm{~h}$. The results were expressed as the mean $\pm \mathrm{SD}$ from three separate experiments.

Change of cell cycle distribution in the Cav-1-knocked down cells treated with bleomycin. In order to further confirm that knockdown of Cav-1 can affect bleomycin action, cell cycle distributions in the HeLa and $\mathrm{HaCaT}$ cells were analyzed by flow cytometry following knockdown of Cav-1 and treatment with bleomycin for $24 \mathrm{~h}$. As shown in Fig. 5, knockdown of Cav-1 had no effect on cell cycle in HeLa cells, whereas it reduced S-phase accumulation in $\mathrm{HaCaT}$ cells. Cav-1 knockdown followed by $2 \mu \mathrm{M}$ bleomycin treatment reduced $\mathrm{G}_{2} / \mathrm{M}$-phase accumulation from $40.6 \%$ in non-transfected HeLa cells to $17.9 \%$ in the transfected cells. It was in agreement with a previous observation by Linge et al (14). However, $\mathrm{G}_{2} / \mathrm{M}$-phase distribution was slightly reduced in $\mathrm{HaCaT}$ cells following Cav-1 knockdown and bleomycin treatment, compared to non-transfected cells.

Action of bleomycin was affected by other cellular factors. In order to explore other cellular factors affecting action of bleomycin except BLH and Cav-1, we compared HeLa and HepG2 cells in which BLH expression levels were similar (Fig. 6A). MTT assay showed that HepG2 cells were more sensitive to bleomycin than HeLa cells (Fig. 6B). As Cav-1 level was different in the two cell lines, knockdown of Cav-1 in HepG2 cells was performed. There was no further increase of sensitivity to bleomycin after reduction of Cav-1 (Fig. 6C and D). The results again indicate that Cav-1 is irrelevant to bleomycin sensitivity, but there are other cellular factors determining it.

We further examined the relationship between expression level of BLH and sensitivity to bleomycin in the cell lines from human lung and liver. Lower level of BLH was detected in human non-small cell lung cancer A549 cells and human embryo normal lung HPF cells (Fig. 7A). The expression level of BLH in normal embryo HEL liver cells was lower than that in hepatoma HepG2 and normal immortalized liver L02 cells. The level of Cav-1 in normal embryo HEL and HPF cells were
A

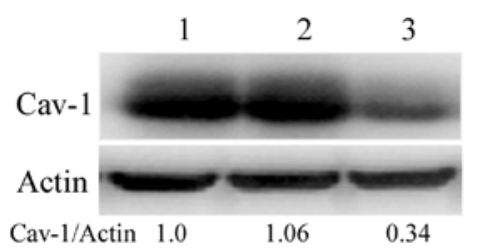

$\mathrm{C}$

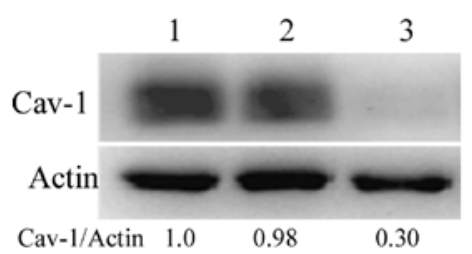

B

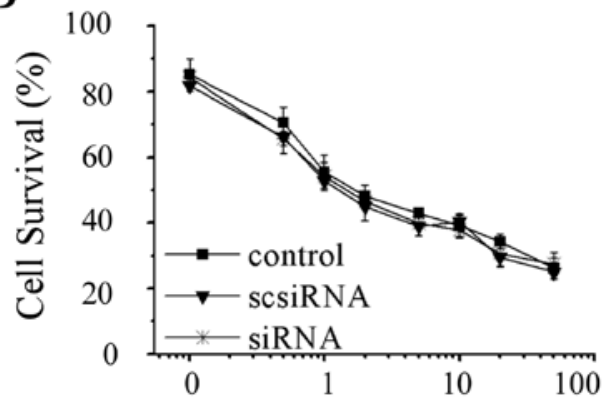

D

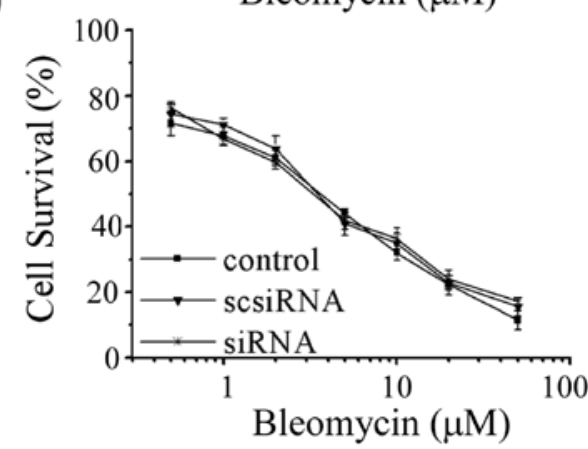

Figure 4. Knockdown of Cav-1 was unrelated with sensitivity to bleomycin. The levels of Cav-1 in HaCaT (A) and HeLa (C) cells were reduced after transfection with siRNA. Lanes 1-3 represent control, scrambled control siRNA and Cav-1 siRNA, respectively. The effects on sensitivity to bleomycin by knockdown of Cav-1 are shown in HaCaT (B) and HeLa (D) cells. The cell viability was determined by MTT assay after treatment with bleomycin for $72 \mathrm{~h}$. A representative result of three independent experiments is shown. 
HeLa
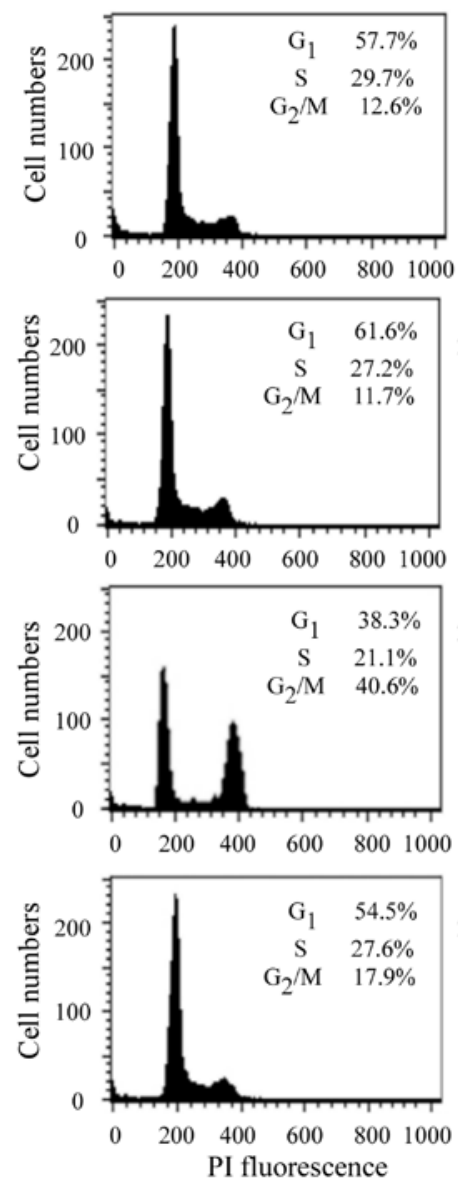

$\mathrm{HaCaT}$
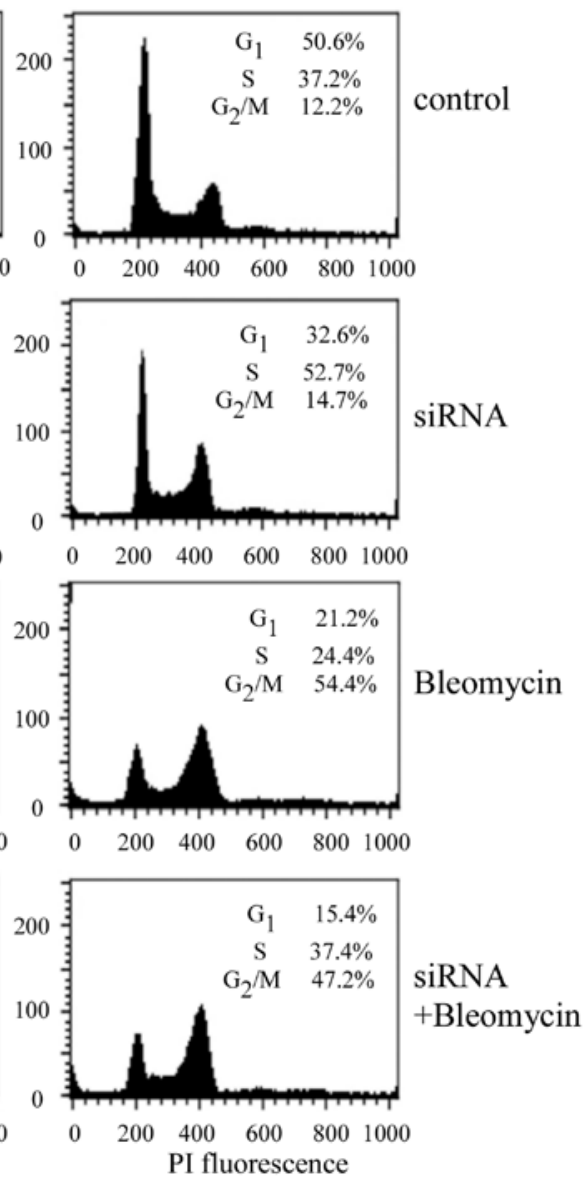

Figure 5. The effects on distribution of cell cycle by knockdown of Cav-1. The cells were subjected to cell cycle analysis after knockdown of Cav-1 with RNA interference and bleomycin treatment for $24 \mathrm{~h}$. The concentrations of bleomycin in HeLa and HaCaT cells were 2 and $0.5 \mu \mathrm{M}$, respectively. A representative result of three independent experiments is shown.

A
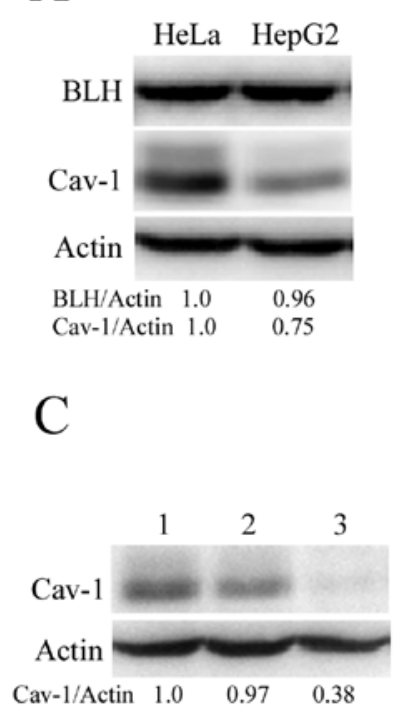

$\mathrm{B}$
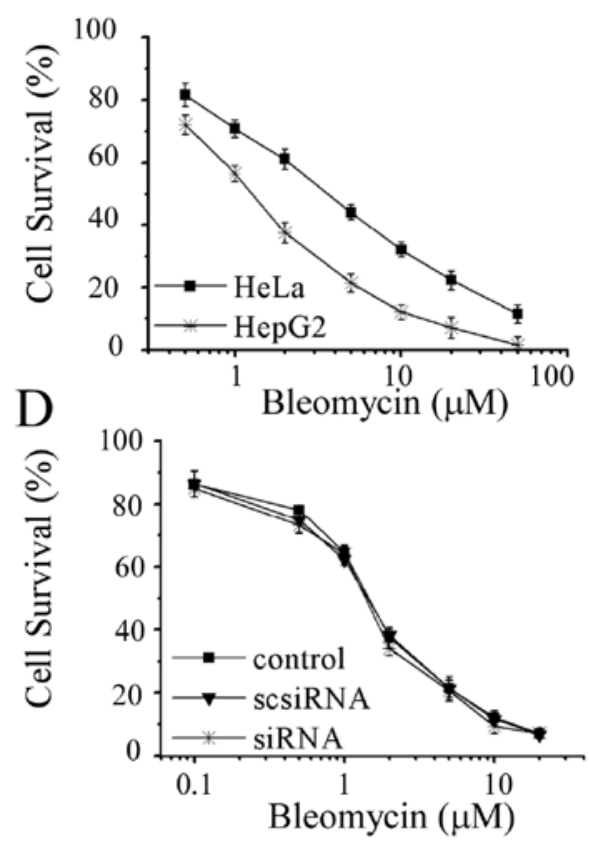

Figure 6. Action of bleomycin was affected by other cellular factors. (A) The levels of BLH and Cav-1 in HeLa and HepG2 cells were detected by western blotting. (B) Sensitivity of HeLa and HepG2 cells toward bleomycin assayed by MTT. (C) The level of Cav-1 in HepG2 cells was knocked down by Cav-1 siRNA. Lane 1, control; lane 2, scrambled control siRNA; lane 3, Cav-1 siRNA. (D) The cell viability was determined by MTT assay after Cav-1 knockdown and treatment with bleomycin for $72 \mathrm{~h}$. The results were expressed as the mean \pm SD from three separate experiments. 

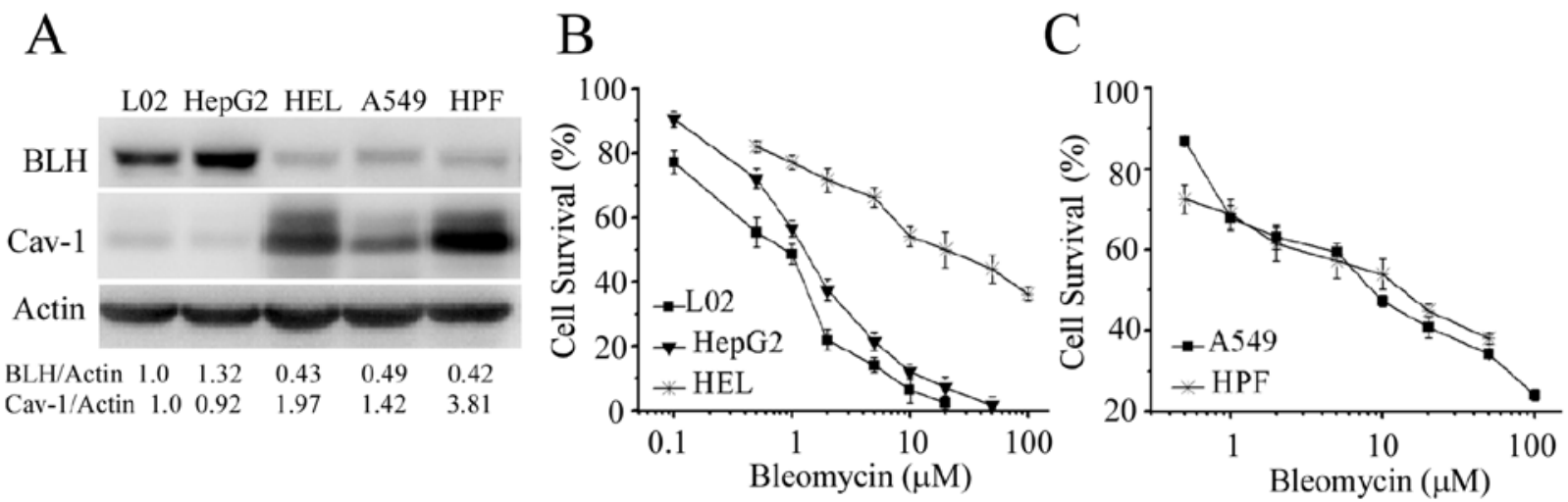

Figure 7. The levels of BLH and sensitivity to bleomycin in the liver- and lung-originated cells. (A) The levels of BLH and Cav-1 in five cell lines were detected by western blotting. (B) Sensitivity of liver-originated L02, HepG2 and HEL cells to bleomycin. (C) Sensitivity of lung-originated A549 and HPF cells to bleomycin. The cell viability was determined by MTT assay after treatment with bleomycin for $72 \mathrm{~h}$. One representative result from three independent experiments is shown.

A

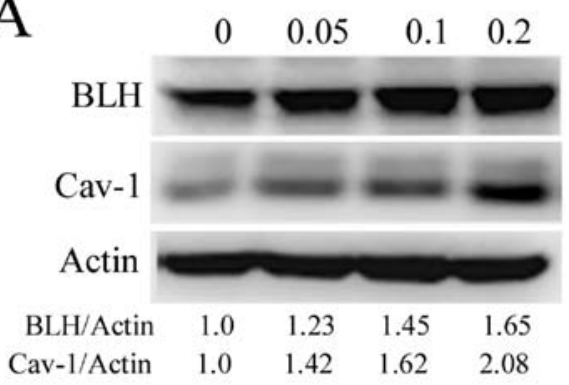

B
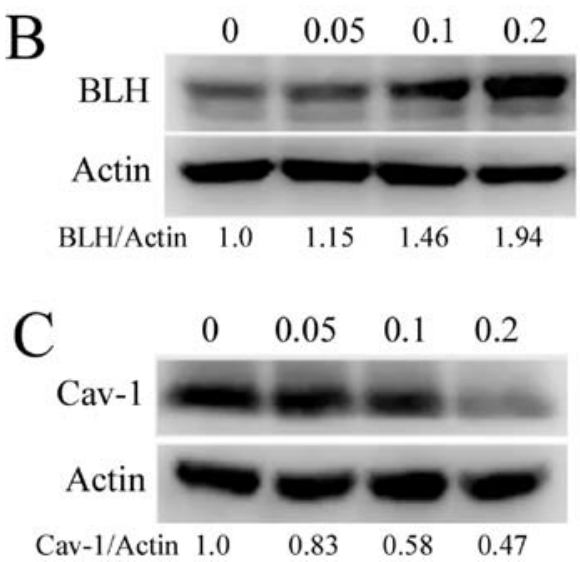

Figure 8. The alterations in BLH and Cav-1 level after bleomycin treatment for $24 \mathrm{~h}$. The levels of BLH and Cav-1 were quantified in comparison with untreated cells [(A) HeLa cells; (B) HepG2 cells; (C) HaCaT cells]. One result from three independent experiments is shown.

higher than that in the other three cell lines. The sensitivity of the five cell types to bleomycin is shown in Fig. 7B and C. Surprisingly, the HEL, A549 and HPF cells containing low BLH level were strikingly more resistant to bleomycin than that in HepG2 and L02 cells. The sensitivity to bleomycin in L02 cells was a little lower than that in HepG2 cells, consistent with the change of BLH level. These findings further supported the notion that besides BLH, other cellular factors could affect the action of bleomycin.
Alterations of BLH and Cav-1 levels after treatment with bleomycin. A previous study indicated that Cav-1 protein levels increased continuously after bleomycin treatment (14). We then determined whether bleomycin treatment influenced the expression levels of Cav-1 and BLH. As shown in Fig. 8A, Cav-1 protein levels increased in a dose-dependent manner after bleomycin treatment at sublethal concentrations for $24 \mathrm{~h}$, consistent with the result reported by Linge et al (14). Unexpectedly, expression of Cav-1 protein in $\mathrm{HaCaT}$ cells gradually decreased (Fig. 8C). The expression levels of BLH in HepG2 and HeLa cells continuously increased in a concentration-dependent manner after treatment with bleomycin for $24 \mathrm{~h}$ (Fig. 8A and B), suggesting that BLH is a response protein.

\section{Discussion}

In this study, we presented evidence to further support the notion that BLH confers resistance to bleomycin. To our knowledge, it is first report that knockdown of BLH markedly increased the sensitivity to bleomycin in HeLa cells. Combining previous reports with our present results, BLH is bona fide biomarker for determination of bleomycin action. The conclusion is based on the following evidence. First, high level of BLH in HL-60 cells was closely related with the high resistance to bleomycin, whereas lower level of BLH in $\mathrm{HaCaT}$ cells was the most sensitive (Fig. 1). Second, BLH expression could be induced by incubation with bleomycin at sublethal concentrations in HeLa and HepG2 cells which contained moderate amount of BLH (Fig. 8), suggesting that BLH can respond to bleomycin treatment. Third, in clinic chemotherapy, the tumors with low level of BLH such as carcinoma of head and neck and lymphoma and Hodgkin's lymphomas achieve good response to bleomycin treatment $(1,17)$. Fourth, the homozygous variant G/G of BLH gene SNP A1450G is associated with reduced survival in testicular gem cell cancer (18). However, attention should be paid to the complicating situations in which lower level of BLH does not mean more sensitive to bleomycin due to effects of other cellular factors. 
Low expression of BLH was observed in A549 and embryo lung fibroblast cells and keratinocyte $\mathrm{HaCaT}$ cells, consistent with previous reports $(2,19,20)$. Unexpectedly, A549 and HPF cells have less sensitive to bleomycin (Fig. 7C), suggesting that $\mathrm{BLH}$ is not participating in bleomycin action in lung cells. According to the clinical reports, up to $46 \%$ of patients will develop pneumonitis and mortality rate is approximately $3 \%$ after administration of bleomycin $(21,22)$, showing individual differences. Therefore, lower level of BLH is not a unique factor to determine the bleomycin-induced pulmonary fibrosis. Several key molecules such as cytosolic phospholipase A2, prostaglandin $\mathrm{F} 22_{2 \alpha}$ receptor, discoidin domain receptor 1 and Cav-1, are reported to be involved in bleomycin-induced pulmonary fibrosis (16,23-25). Knockdown of Nrf-2 obviously augmented cytotoxicity of bleomycin to A549 cells (26). It is clear that other cellular factors except BLH determine cytotoxicity of bleomycin to lung cells.

In liver, the relationship between expression level of BLH and sensitivity to bleomycin is very complicated. Our results showed more sensitivity to bleomycin but BLH level is high in the HepG2 cells (Fig. 7A). In an earlier study, 700-fold survival difference in five rat hepatoma cell lines was observed after treatment with bleomycin (27). Although BLH gene was identified as a methylated tumor suppressor gene in hepatocellular carcinoma, only half of the patients displayed lower BLH expression in hepatocellular carcinoma tissues (28). What determines the different sensitivity to bleomycin in hepatoma and normal cells require further investigation.

In the present results, we have shown that the BLH levels augmented after exposure to bleomycin at sublethal concentrations. It is helpful to explain the reason why induction of bleomycin resistance led to increased BLH activity (29,30). BLH may be a response protein to resist cytotoxicity of bleomycin. However, the BLH activity in bleomycin-resistant sublines was not consistent with the resistance level to bleomycin in human head and neck squamous cell carcinoma A-253 cell line (29), suggesting that BLH is not mainly involved in resistance to bleomycin. Therefore, BLH may play an important role in natural resistance to primary exposure of bleomycin in certain types of cell lines. Several studies reported that Cav-1 expression was involved in the resistance to some anticancer drugs $(31,32)$. The likelihood that Cav-1 mediates sensitivity to bleomycin is ruled out by our present data.

Cav-1, a principal component of caveolae, is found in most mammalian cells including adipocytes, endothelial cells and fibroblasts (33). An important role of caveolin-1 in cellular senescence is demonstrated in vitro by overexpression of Cav-1 in young human diploid fibroblasts and primary cultures of murine fibroblasts $(34,35)$. Oxidative stress induces premature senescence by stimulating caveolin-1 gene transcription through $\mathrm{p} 38$ mitogen-activated protein kinase/SP-1 mediated activation of Cav-1 promoter elements (36). Knockdown of Cav-1 can reverse senescent human fibroblasts to re-enter the cell cycle (37). In our experiments, the results with increased level of Cav-1 and disruption of cell cycle distribution after knockdown of Cav-1 are in agreement with a previous study (14). Accumulating data showed that bleomycin can induce cellular senescence in many cell lines (38-40). All the evidence indicate that the role of Cav-1 expression after treatment with bleomycin is to maintain the morphological feature of cellular senescence.
The sensitivity to bleomycin is also mediated by transported proteins in cell membrane. L-carnitine transporter hCT2 was responsible for bleomycin-A5 uptake (17). In the cell lines we studied, the expression of hCT2 mRNA was not detected by RT-PCR (data not shown), ruling out its involvement in bleomycin action. Accumulation data showed that resistance to bleomycin was due to repair enzymes, overexpression of antioxidant enzymes and antioxidant Q10 $(30,41)$. Evaluating all factors reported for prediction of sensitivity to bleomycin is under way in our laboratory.

$\mathrm{BLH}$, a neutral cysteine protease, is associated with other diseases including atopic dermatitis, keratinization disorders and Alzheimer's disease (42-44). It also plays an important role in antigen presentation, hydrolysis of homocysteinethiolactone, breakdown of deiminated filaggrin into amino acids N-terminal proteolysis of huntingtin (45-48). Exploring the function of BLH in this study will benefit translational research also in other diseases.

In conclusion, the present data demonstrate that BLH is indeed involved in modulation of bleomycin action, but Cav-1 is not associated with it. It is valuable to take BLH as one of the biomarkers for prediction of individual tumor response to bleomycin treatment. Combining other cellular factors with BLH will more exactly predict the sensitivity to bleomycin and track the tumor responses towards new antibiotics of bleomycin family in clinic trials.

\section{Acknowledgements}

This study was supported by grants from the National S\&T Major Special Project on Major New Drug Innovation (2012ZX09301002-001), State Mega programs (2010ZX09401403) and National Scientific Foundation of China (81273553).

\section{References}

1. Chen J and Stubbe J: Bleomycins: towards better therapeutics. Nat Rev Cancer 5: 102-112, 2005.

2. Lazo JS and Humphreys CJ: Lack of metabolism as the biochemical basis of bleomycin-induced pulmonary toxicity. Proc Natl Acad Sci USA 80: 3064-3068, 1983

3. Ibrahimi OA and Anderson RR: Images in clinical medicine. Bleomycin-induced flagellate hyperpigmentation. N Engl J Med 363: e36, 2010.

4. Xu H, Yu L, Zhang X and Wang S: Isolation, purification and structure determination of boningmycin (Z-893). J Chin Antibiot 28: 465-467, 2003

5. Gao N, Shang B, Zhang X, et al: Potent antitumor actions of the new antibiotic boningmycin through induction of apoptosis and cellular senescence. Anticancer Drugs 22: 166-175, 2011.

6. Lazo JS, Boland CJ and Schwartz PE: Bleomycin hydrolase activity and cytotoxicity in human tumors. Cancer Res 42: 4026-4031, 1982.

7. Schwartz DR, Homanics GE, Hoyt DG, Klein E, Abernethy J and Lazo JS: The neutral cysteine protease bleomycin hydrolase is essential for epidermal integrity and bleomycin resistance. Proc Natl Acad Sci USA 96: 4680-4685, 1999.

8. Pei Z, Calmels TP, Creutz CE and Sebti SM: Yeast cysteine proteinase gene ycpl induces resistance to bleomycin in mammalian cells. Mol Pharmacol 48: 676-681, 1995.

9. Lazo JS, Merrill WW, Pham ET, Lynch TJ, McCallister JD and Ingbar DH: Bleomycin hydrolase activity in pulmonary cells. J Pharmacol Exp Ther 231: 583-588, 1984.

10. Sebti SM, Jani JP, Mistry JS, Gorelik E and Lazo JS: Metabolic inactivation: a mechanism of human tumor resistance to bleomycin. Cancer Res 51: 227-232, 1991

11. Wang H and Ramotar D: Cellular resistance to bleomycin in Saccharomyces cerevisiae is not affected by changes in bleomycin hydrolase levels. Biochem Cell Biol 80: 789-796, 2002. 
12. Einhorn LH: Curing metastatic testicular cancer. Proc Natl Acad Sci USA 99: 4592-4595, 2002.

13. Aouida M, Poulin R and Ramotar D: The human carnitine transporter SLC22A16 mediates high affinity uptake of the anticancer polyamine analogue bleomycin-A5. J Biol Chem 285 : 6275-6284, 2010.

14. Linge A, Weinhold K, Bläsche R, Kasper M and Barth K: Downregulation of caveolin-1 affects bleomycin-induced growth arrest and cellular senescence in A549 cells. Int J Biochem Cell Biol 39: 1964-1974, 2007.

15. Linge A, Meleady P, Henry M, Clynes M, Kasper M and Barth K Bleomycin treatment of A549 human lung cancer cells results in association of MGrl-Ag and caveolin-1 in lipid rafts. Int J Biochem Cell Biol 43: 98-105, 2011

16. Wang XM,Zhang Y, Kim HP, et al: Caveolin-1: a critical regulator of lung fibrosis in idiopathic pulmonary fibrosis. J Exp Med 203: 2895-2906, 2006.

17. Ferrando AA, Velasco G, Campo E and Lopez-Otin C: Cloning and expression analysis of human bleomycin hydrolase, a cysteine proteinase involved in chemotherapy resistance. Cancer Res 56 $1746-1750,1996$

18. de Haas EC, Zwart N, Meijer C, et al: Variation in bleomycin hydrolase gene is associated with reduced survival after chemotherapy for testicular germ cell cancer. J Clin Oncol 26: 1817-1823, 2008 .

19. Brömme D, Rossi AB, Smeekens SP, Anderson DC and Payan DG: Human bleomycin hydrolase: molecular cloning, sequencing, functional expression, and enzymatic characterization. Biochemistry 35: 6706-6714, 1996

20. Sikic B: Biochemical and cellular determinants of bleomycin cytotoxicity. Cancer Surv 5: 81-91, 1986.

21. Sleijfer S: Bleomycin-induced pneumonitis. Chest 120: 617-624 2001.

22. Simpson AB, Paul J, Graham J and Kaye SB: Fatal bleomycin pulmonary toxicity in the west of Scotland 1991-95: a review of patients with germ cell tumours. Br J Cancer 78: 1061-1066, 1998

23. Nagase T, Uozumi N, Ishii S, et al: A pivotal role of cytosolic phospholipase A(2) in bleomycin-induced pulmonary fibrosis. Nat Med 8: 480-484, 2002

24. Oga T, Matsuoka T, Yao C, et al: Prostaglandin F(2alpha) receptor signaling facilitates bleomycin-induced pulmonary fibrosis independently of transforming growth factor-beta. Nat Med 15: $1426-1430,2009$

25. Avivi-Green C, Singal M and Vogel WF: Discoidin domain receptor 1-deficient mice are resistant to bleomycin-induced lung fibrosis. Am J Respir Crit Care Med 174: 420-427, 2006.

26. Homma S, Ishii Y, Morishima Y, et al: Nrf2 enhances cell proliferation and resistance to anticancer drugs in human lung cancer. Clin Cancer Res 15: 3423-3432, 2009.

27. Barranco SC, Haenelt BR and Gee EL: Differential sensitivities of five rat hepatoma cell lines to anticancer drugs. Cancer Res 38: 656-660, 1978

28. Okamura Y, Nomoto S, Hayashi M, et al: Identification of the bleomycin hydrolase gene as a methylated tumor suppressor gene in hepatocellular carcinoma using a novel triple-combination array method. Cancer Lett 312: 150-157, 2011.

29. Lazo JS, Braun ID, Labaree DC, Schisselbauer JC, Meandzija B, Newman RA and Kennedy KA: Characteristics of bleomycinresistant phenotypes of human cell sublines and circumvention of bleomycin resistance by liblomycin. Cancer Res 49: 185-190, 1989.

30. Yen HC, Li SH, Majima HJ, et al: Up-regulation of antioxidant enzymes and coenzyme $\mathrm{Q}(10)$ in a human oral cancer cell line with acquired bleomycin resistance. Free Radic Res 45: 707-716, 2011.
31. Belanger MM, Roussel E and Couet J: Up-regulation of caveolin expression by cytotoxic agents in drug-sensitive cancer cells. Anticancer Drugs 14: 281-287, 2003.

32. Tirado O, MacCarthy C, Fatima N, Villar J, Mateo-Lozano S and Notario V: Caveolin-1 promotes resistance to chemotherapyinduced apoptosis in Ewing's sarcoma cells by modulating PKCalpha phosphorylation. Int J Cancer 126: 426-436, 2010.

33. Parton RG and Simons K: The multiple faces of caveolae. Nat Rev Mol Cell Biol 8: 185-194, 2007.

34. Park WY, Park JS, Cho KA, Kim DI, Ko YG, Seo JS and Park SC Up-regulation of caveolin attenuates epidermal growth factor signaling in senescent cells. J Biol Chem 275: 20847-20852, 2000.

35. Volonte D, Zhang K, Lisanti MP and Galbiati F: Expression of caveolin-1 induces premature cellular senescence in primary cultures of murine fibroblasts. Mol Biol Cell 13: 2502-2517, 2002.

36. Dasari A, Bartholomew JN, Volonte D and Galbiati F: Oxidative stress induces premature senescence by stimulating caveolin-1 gene transcription through $\mathrm{p} 38$ mitogen -activated protein kinase/ Spl-mediated activation of two GC-rich promoter elements. Cancer Res 66: 10805-10814, 2006.

37. Cho KA, Ryu SJ, Park JS, Jang IS, Ahn JS, Kim KT and Park SC: Senescent phenotype can be reversed by reduction of caveolin status. J Biol Chem 278: 27789-27795, 2003.

38. Robles SJ and Adami GR: Agents that cause DNA double strand breaks lead to $16^{\mathrm{INK} 4 \mathrm{a}}$ enrichment and the premature senescence of normal fibroblasts. Oncogene 16: 1113-1123, 1998.

39. Aoshiba K, Tsuji T and Nagai A: Bleomycin induces cellular senescence in alveolar epithelial cells. Eur Respir J 22: 436-443, 2003.

40. Baus F, Gire V, Fisher D, Piette J and Dulić V: Permanent cell cycle exit in $\mathrm{G} 2$ phase after DNA damage in normal human fibroblasts. EMBO J 22: 3992-4002, 2003.

41. Robertson KA, Bullock HA, Xu Y, et al: Altered expression of Ape1/ref-1 in germ cell tumors and overexpression in NT2 cells confers resistance to bleomycin and radiation. Cancer Res 61: 2220-2225, 2001

42. Kamata Y, Yamamoto M, Kawakami F, Tsuboi R, Takeda A, Ishihara $\mathrm{K}$ and Hibino T: Bleomycin hydrolase is regulated biphasically in a differentiation- and cytokine-dependent manner: relevance to atopic dermatitis. J Biol Chem 286: 8204-8212, 2011.

43. Kamata Y, Maejima H, Watarai A, Saito N, Katsuoka K, Takeda A and Ishihara K: Expression of bleomycin hydrolase in keratinization disorders. Arch Dermatol Res 304: 31-38, 2012.

44. Suszynska J, Tisonczyk J, Lee HG, Smith MA and Jakubowski H: Reduced homocysteine-thiolactonase activity in Alzheimer's disease. J Alzheimers Dis 19: 1177-1183, 2010.

45. Towne CF, York IA, Watkin LB, Lazo JS and Rock KL: Analysis of the role of bleomycin hydrolase in antigen presentation and the generation of CD8 T cell responses. J Immunol 178: 6923-6930, 2007.

46. Kamata Y, Taniguchi A, Yamamoto M, et al: Neutral cysteine protease bleomycin hydrolase is essential for the breakdown of deiminated filaggrin into amino acids. J Biol Chem 284: 12829-12836, 2009.

47. Zimny J, Sikora M, Guranowski A and Jakubowski H: Protective mechanisms against homocysteine toxicity: the role of bleomycin hydrolase. J Biol Chem 281: 22485-224892, 2006.

48. Ratovitski T, Chighladze E, Waldron E, Hirschhorn RR and Ross CA: Cysteine proteases bleomycin hydrolase and cathepsin Z mediate $\mathrm{N}$-terminal proteolysis and toxicity of mutant huntingtin. J Biol Chem 286: 12578-12589, 2011. 